European Journal of Pharmaceutical Sciences Volume 29, Issues 3-4, November 2006, Pages 315-324 Advances in Understanding Oral Absorption and Delivery of Problem Compounds - Selected Papers from the 3rd World Conference on Drug Absorption, Transport and Delivery

doi:10.1016/j.ejps.2006.05.001 (2) Citz or Link Using DGl

Copyright (C) 2006 Elsevier B.V. All rights reserved.

\title{
Review
}

\section{Review of global regulations concerning biowaivers for immediate release solid oral dosage forms ${ }^{\text {th }}$}

\section{E. Gupta ${ }^{a}$, D.M. Barends ${ }^{\text {b, }}$, , E. Yamashitaa ${ }^{a}$ K.A. Lentz ${ }^{c}$, A.M. Harmsze ${ }^{d}$, V.P. Shahe, J.B. Dressman ${ }^{f}$ and R.A. Lipper}

${ }^{a}$ Bristol-Myers Squibb Pharmaceutical Research Institute, Princeton, NJ, USA ${ }^{\mathrm{b}}$ RIVM - National Institute for Public Health and the Environment, P.O. Box 1, 3720 BA Bilthoven, The Netherlands

${ }^{\circ}$ Bristol-Myers Squibb Pharmaceutical Research Institute, Wallingford, CT, USA

${ }^{\mathrm{d}}$ St. Antonius Hospital, Department of Clinical Pharmacy, Nieuwegein, The Netherlands

ePharmaceutical consultant, North Potomac, MD 20878, USA

${ }^{f}$ Institute of Pharmaceutical Technology, Biocenter, J.W. Goethe University, Frankfurt am Main, Germany

This paper reflects the scientific opinion of the authors and not the policies of regulating agencies.

* Corresponding author. Tel.: +31 30 2744209; fax: +31 302744462.

\begin{abstract}
The regulations with respect to biowaivers for immediate release (IR) solid oral dosage forms in the USA, the EU, Japan and from the World Health Organization (WHO) are summarized and compared. Two case studies are presented, one from our own files and one from the open literature, showing the similarities and the differences among the qualification requirements of the four systems. The regulatory experience gained up to now is reviewed and expected future trends are discussed.
\end{abstract}

Keywords: Biopharmaceutics Classification System (BCS); Regulations

\section{Introduction}

In vivo bioequivalence ( $\mathrm{BE}$ ) assessment of solid oral dosage forms is a commonly accepted surrogate for judging therapeutic equivalence of pharmaceutically equivalent drug products, which offers the advantage of obviating the need for additional clinical evaluation. But current research on the impact of composition and manufacture of a drug product on drug absorption in relation to the biopharmaceutical properties of the active pharmaceutical ingredient (API) has led to the acceptance of in vitro testing as a sufficiently reliable surrogate for an in vivo BE study for certain drug products. The regulatory acceptance of in vitro testing as a reliable surrogate for an in vivo BE study is commonly referred to as 
European Journal of Pharmaceutical Sciences Volume 29, Issues 3-4, November 2006, Pages 315-324 Advances in Understanding Oral Absorption and Delivery of Problem Compounds - Selected Papers from the 3rd World Conference on Drug Absorption, Transport and Delivery

"biowaiver". This review presents an overview of the global regulatory guidances for biowaiving to the following categories of formulation development:

(A) Formulation development for a new drug product. This is an evolutionary process with inevitable changes in composition, components and manufacturing processes. Resulting differences in formulation and/or manufacturing between proof-of-principle (Phase II) formulations, pivotal formulations (Phase III) and long-term stability test formulations have to be qualified, i.e. their BE to the ultimate commercial formulation has to be demonstrated. In addition, blinded comparators used in pivotal clinical studies have to be qualified.

(B) Line extensions, e.g., new strengths or new formulations for a new patient population, for instance, a pediatric formulation.

(C) Formulation development of a generic drug product. A generic drug product must pharmaceutically be equivalent ànd bioequivalent to an innovator drug product in order to be therapeutically equivalent to and interchangeable with that innovator drug product.

(D) Post-approval changes both for innovator and generic drug products. These include changes of the manufacturing formula, in the manufacturing process, in excipients, in manufacturing sites and/or equipment. All these changes necessitate demonstration of BE and may be eligible for the biowaiver option in certain cases.

\section{Regulations in the USA}

The present USA guidances that consider biowaivers are:

- Immediate release solid oral dosage forms. Scale-up and post-approval changes (FDA, SUPAC-IR, 1995).

- Extended release oral dosage forms: development, evaluation and application of in vitro/in vivo correlations (FDA, IVIVC, 1997).

- Waiver of in vivo bioavailability (BA) and bioequivalence studies for immediate release solid oral dosage forms based on a Biopharmaceutics Classification System (FDA, BCS, 2000).

- Bioavailability and bioequivalence studies for orally administered drug productsgeneral considerations (FDA, BA/BE, 2003).

\subsection{FDA, SUPAC-IR (1995)}

The guidance FDA, SUPAC-IR (1995) was the first regulatory document to establish a consistent and clear policy for scale-up and post-approval changes to IR drug products, allowing qualification of several types of changes in composition and manufacturing process, with specific requirements based on the "level" of change. The likely impacts of changes on formulation quality and performance are categorized as Level 1 (minimal impact), Level 2 (could have a significant impact) or Level 3 (likely to have a significant impact). Types of post-approval changes in composition, manufacturing site, batch size and manufacturing process with respect to dissolution and $B E$ requirements are summarized in Table 1 . Most Level 3 changes can only be qualified by in vivo BE, but many Level 1 and 2 changes can be qualified by in vitro dissolution. Level 1 requires only a demonstration that 
European Journal of Pharmaceutical Sciences Volume 29, Issues 3-4, November 2006, Pages 315-324 Advances in Understanding Oral Absorption and Delivery of Problem Compounds - Selected Papers from the 3rd World Conference on Drug Absorption, Transport and Delivery

the drug product after the changes still complies with the in vitro dissolution release specification. Level 2 requirements differ depending on the therapeutic range, solubility, and permeability. Multiple media testing and compliance of the similarity of the dissolution profiles to the $f_{2}$ criterion are needed, depending on the solubility and permeability determinations. Drug products containing an API that is "highly soluble" and "highly permeable and showing $\geq 85 \%$ dissolution in $900 \mathrm{~mL} 0.1 \mathrm{~N} \mathrm{HCl}$ within $15 \mathrm{~min}$ are exempted from the dissolution similarity requirements. "High solubility" of the API is defined as highest dosage strengthto-solubility ratio being $\leq 250 \mathrm{~mL}$ over a $\mathrm{pH}$ range of $1-8$, and "highly permeable" if the extent of in vivo oral absorption is $\geq 90 \%$. A caveat to this regulation is that post-approval changes for drug products containing APIs having a narrow therapeutic index (NTI) always require in vivo BE qualification. The guidance FDA, SUPAC-IR (1995) does not define what is understood by a NTI, but the US Code of Federal Regulations (CFR, 2005) defines it as "less than a two-fold difference in minimum toxic concentrations and minimum effective concentrations in the blood; and safe and effective use of the drug products requires careful dosage titration and patient monitoring". Qualification for post-approval changes not identified in the guidance FDA, SUPAC-IR (1995) have to be determined on a case-by-case basis, based on justification of the impact of the change on formulation performance.

\subsection{FDA, BCS (2000)}

The guidance FDA, BCS (2000) categorizes APIs into four classes:

Class 1: High solubility-high permeability.

Class 2: Low solubility-high permeability.

Class 3: High solubility-low permeability.

Class 4: Low solubility-low permeability.

In the guidance FDA, BCS (2000), the highest dosage strength-to-solubility ratio according to the guidance FDA, SUPAC-IR (1995) is redefined with respect to the $\mathrm{pH}$ range, which is reduced to $\mathrm{pH} 1-7.5$ but must include solubility data at $\mathrm{pH}=\mathrm{p} K_{\mathrm{a}}$ and $\mathrm{pH}=\mathrm{p} K_{\mathrm{a}} \pm 1$.

Guidance FDA, BCS (2000) allows biowaivers for drug products with BCS Class 1 APIs, i.e., APIs exhibiting high solubility and high permeability. The guidance does not accept biowaivers for drug products containing APIs belonging to BCS Class 2, 3 and 4 . In addition, the guidance sets requirements for the drug product, in that it must exhibit rapid dissolution, defined as at least $85 \%$ of the dose being dissolved within $30 \mathrm{~min}$ in $900 \mathrm{~mL}$ or less of simulated gastric fluid or $0.1 \mathrm{~N} \mathrm{HCL}$, in $\mathrm{pH} 4.5$ buffer, and in simulated intestinal fluid or $\mathrm{pH} 6.8$ buffer.

Further criteria that have to be met to qualify for a biowaiver are:

(i) The API may not be an NTI.

(ii) The excipients should not affect rate and extent of drug absorption. This criterion is considered to be met when the excipients present in the drug product are also present in FDA-approved IR solid oral dosage forms in a quantity consistent with the intended function. 
European Journal of Pharmaceutical Sciences Volume 29, Issues 3-4, November 2006, Pages 315-324 Advances in Understanding Oral Absorption and Delivery of Problem Compounds - Selected Papers from the 3rd World Conference on Drug Absorption, Transport and Delivery

(iii) The drug product is not intended to be absorbed in the oral cavity.

(iv) The API may not be a prodrug.

If all these conditions are met, dissolution similarity between test and reference drug product in the three media based on the criterion that $f_{2} \geq 50$ are sufficient to qualify the test drug product for a biowaiver. Should both the test and reference drug product show $\geq 85 \%$ dissolution within 15 min in each of the three media, then the test product qualifies for a biowaiver without the need to apply the $f_{2}$ criterion for similarity of the dissolution profiles.

In essence, guidance FDA, BCS (2000) serves as an extension of the guidance FDA, SUPAC-IR (1995), by providing a mechanism for qualifying biowaivers for APIs with high permeability and high solubility, irrespective of the type or level of change.

\subsection{FDA, BA/BE (2003)}

The guidance FDA, BA/BE (2003) provided yet another option for in vitro qualification of lower/higher strengths of drug products, based on 'proportional similarity' between the strengths. There are three options to qualify a formulation as "proportionally similar" to another formulation:

(i) when a common granulation is used in the preparation of the various strengths.

(ii) if the sum of all differences in excipients between the two formulations is within $10 \%$, i.e. falls within Level 2 of guidance FDA, SUPAC-IR (1995).

(iii) for high potency APIs ( $\leq 5 \mathrm{mg}$ dose), when the total weight of the two strengths is the same, the same excipients are used and the small difference in composition is compensated by one of the excipients.

\subsection{FDA, IVIVC (1997)}

The guidance FDA, IVIVC (1997) addresses modified release (MR) formulations and hence falls outside the scope of this review concerning IR solid oral dosage forms. However, since an IVIVC can exist if in vivo dissolution is the rate-limiting step in the absorption, IR solid oral dosage forms containing a BCS Class 2 API can show an IVIVC as well as MR formulations. And if an IVIVC can be developed, this could also result in a biowaiver. However, IVIVCs for IR solid oral dosages forms are seldom established and hence not further discussed in this review.

\section{Regulations in the EU}

The system of the EU notes for guidance is not fully identical to the system of the FDA guidances, so, not all FDA guidances have a counterpart in the EU regulations and vice versa. The EU does not have separate regulatory documents on BCS and biowaivers. Biowaiving in general and biowaiving per BCS are covered both in the note for guidance on the investigation of bioavailability and bioequivalence (EMEA, BA/BE, 2001). This guidance is applicable to new applications and to post-approval changes; in the EU post-approval changes are called variations. The guidance EMEA, BA/BE (2001) also describes dose proportionality and the possibilities and requirements for biowaiving in those situations. 
European Journal of Pharmaceutical Sciences Volume 29, Issues 3-4, November 2006, Pages 315-324 Advances in Understanding Oral Absorption and Delivery of Problem Compounds - Selected Papers from the 3rd World Conference on Drug Absorption, Transport and Delivery

The regulations with respect to variations in marketing authorizations (MAs) in the EU system are also not structured in the same way as in the guidance FDA, SUPAC-IR (1995). In the Notice to Applicants: Guideline on dossier requirements for Type IA and Type IB notifications (EU, Variations, 2003), the EU defines 47 "small" variations called Type I variations; "larger" variations are called Type II variations. Type I and Type II differ in their regulatory procedure, such as time limits, rather than in the technical requirements for qualifications to support these changes. The possibility for a biowaiver is given in a number of the Type I variations, but most require a justification for not submitting an in vivo BE study according to the considerations described in the guidance EMEA, BA/BE (2001). For Type II variations biowaiving also may be acceptable, but again requires a justification for not submitting an in vivo BE study according to the guidance, see Table 2. Thus, in the EU system there are only a few variations that are predefined deemed acceptable for a biowaiver. In the EU, proportional similarity is referred to as dose proportionality and is defined somewhat differently than by the FDA. According to the EU regulations, the ratio between the amounts of the API and the excipients must be the same, or, when the concentration of API is $<5 \%$, the ratio between the excipients has to be similar. So, small differences in composition between the different strengths as allowed by the guidance FDA, SUPAC-IR (1995) are outside the definition of dose proportionality in the EU regulation. Also, the guidance EMEA, BA/BE (2001) accepts biowaiving only when different strengths are manufactured by the same manufacturer and the same manufacturing process; such a requirement is not found in the FDA regulations.

In the technical requirements for biowaiving there are only very small differences between the FDA and EU, which facilitates adoption of a global approach to developing biowaiver strategies. The differences between the FDA and EU requirements can be summarized as follows.

(i) In the guidance EMEA, BA/BE (2001), an API is defined as "highly soluble" when the amount in the highest dosage form strength can be dissolved in $250 \mathrm{~mL}$ or less of each of three buffers, preferably at or about $1.0,4.6,6.8$, at $37^{\circ} \mathrm{C}$. With respect to the definition of "highly soluble", two small differences exist with the FDA regulations: the FDA requires solubility in a $\mathrm{pH}$-range: $\mathrm{pH} 1-7.5$, instead of $\mathrm{pH} 1-6.8$ and specific solubility data at $\mathrm{pH}=\mathrm{p} K_{\mathrm{a}}$ and $\mathrm{pH}=\mathrm{p} K_{\mathrm{a}} \pm 1$.

(ii) In the EU regulations, there is no quantitative criterion for an API to be "highly permeable". The guidance EMEA, BA/BE (2001) states that "linear and complete absorption indicating high permeability reduces the possibility of an IR dosage form influencing the BA". In contrast, the guidance FDA, BCS (2000) defines an API as "highly permeable" when the extent of absorption in humans is $90 \%$ or more. Also, the guidance FDA, BCS (2000) describes techniques for determining the API permeability class, whereas such a description is not present in the guidance EMEA, BA/BE (2001).

(iii) The guidance EMEA, BA/BE (2001) excludes drug products containing an API with a history of BA-problems and bioinequivalence.

(iv) The guidance EMEA, BA/BE (2001) considers the linearity of the pharmacokinetics of the API for biowaivers based on the BCS, see (ii). For biowaivers based on dose proportionality, both regulatory systems require linear pharmacokinetics.

(v) The guidance EMEA, BA/BE (2001) requires addressing the method of manufacture of the drug products in relation to the critical physicochemical properties (particle size, polymorphic form) of the API. 
European Journal of Pharmaceutical Sciences Volume 29, Issues 3-4, November 2006, Pages 315-324 Advances in Understanding Oral Absorption and Delivery of Problem Compounds - Selected Papers from the 3rd World Conference on Drug Absorption, Transport and Delivery

\section{Regulations in Japan}

For the first approval of a generic IR solid oral drug product in Japan, no biowaiving is accepted under Japanese regulations; in vivo $\mathrm{BE}$ is always required (NIHS, 1997 and Aoyagi, 2000). Japanese guidances on BA and BE relate to qualification of post-approval changes in components and composition of drug products (NIHS, 2000a) as well as for products with strengths that differ from the 'approved' product (NIHS, 2000b). The qualification strategies outlined in these guidances are also applicable to changes in generic drug products post-approval. The guideline for BE studies for formulation changes of oral solid dosage forms identifies different requirements for qualification based on the magnitude of the change, on the therapeutic index of the API, on the dissolution of the drug product and on the solubility of the API. The changes are categorized as Levels AD. Biowaivers are not granted for any drug products containing a low solubility API and only for drug products containing a NTI API provided (a) the change is small, (b) the API's solubility is not low and (c) the drug product dissolves $>85 \%$ in multiple media within $30 \mathrm{~min}$. Unlike the FDA, the Japanese regulations allow in vitro qualification for a larger formulation change as long as the API is a non-NTI API and provided $\geq 85 \%$ dissolves in $30 \mathrm{~min}$ in multiple media. The draft Japanese guidance relating to $\mathrm{BE}$ studies for manufacturing changes for conventional and enteric coated solid oral dosage forms (Aoyagi et al., 2004) has been published recently and is aligned with the guidance FDA, SUPAC-IR (1995).

The Japanese guidelines for addressing formulation changes seem thus to be aligned in several respects with the guidance FDA, SUPAC-IR (1995), and hence with the EU guidelines. However, some differences are highlighted below:

(i) Levels $A-C$ in the Japanese guideline correspond with the guidance FDA, SUPAC-IR (1995), Levels 1-3. However, there is also a Level D change, specific to Japan, allowing for a larger change for a drug product that however is not expected to result in significant bioinequivalence problems. The Level $D$ change is similar to the Level 3 of the guidance FDA, SUPAC-IR (1995), but allows for biowaiver of drug products that have a rapid dissolution and contain a non-low solubility and non-NTI API.

(ii) There can be separate qualification requirements for core versus coating layer for coated products. Levels B-D allow for 5, 10 and 15\% in total change of coating agents and weight.

(iii) Lack of recognition of a BCS-based biowaivers, i.e. based on characteristics of the API. It is believed that differences in BA between test and reference products are driven by formulation changes that are not addressed by the solubility and permeability characteristics of the API.

Table $3 \mathrm{~A}$ and $\mathrm{B}$ summarize composition and manufacturing changes and biowaiver options under the Japanese regulations.

\section{World Health Organization (WHO) guidelines}

The WHO is not a regulatory body, but it publishes technical reports and guidances which are recommendations to national authorities. Many national authorities give these WHO reports national regulatory status. The WHO documents primarily focus on the needs of developing countries but also endeavor to reflect the state-of-the-art. 
European Journal of Pharmaceutical Sciences Volume 29, Issues 3-4, November 2006, Pages 315-324 Advances in Understanding Oral Absorption and Delivery of Problem Compounds - Selected Papers from the 3rd World Conference on Drug Absorption, Transport and Delivery

The WHO guidance: Multisource (generic) pharmaceutical products: guidelines on registration requirements to establish interchangeability (WHO, Multisource, 1996) deals only with registration requirements of generic medicinal products only, which are described by WHO as "multisource pharmaceutical products". This guidance also defines dose-proportionality. There is no separate WHO document on registration requirements for post-approval changes.

The guidance WHO, Multisource (1996) does not mention biowaivers. However, very recently, this guidance has been revised (WHO, Multisource, 2005a). This revision is not yet published, but in the revised guideline, biowaivers based on $\mathrm{BCS}$ are introduced prominently, recommending more relaxed criteria than the present FDA and EU regulations, both of which limit biowaivers to BCS Class 1, suggesting that the FDA and EU biowaiver criteria are rather conservative and that it is appropriate to broaden its application to well-known APIs such as those on the Model List of Essential Medicines List (WHO, 2005b).

In addition to the guidance WHO, Multisource (2005a), the WHO is in the process of adopting a new guidance: Proposal to waive in vivo BE requirements for WHO Model List of Essential Medicines IR solid oral dosage forms (WHO, Biowaiver, 2000c). For the APIs on the list, the guidance WHO, Biowaiver (2005a), provides information to national authorities relevant to the decision as to whether a biowaiver can be granted or not, taking into account the physical-chemical parameters of that API as summarized below, its therapeutic indication and therapeutic window.

The differences of the guidance WHO, Biowaiver (2000c) with the FDA requirements are:

(i) Consideration if an API has a NTI or not has been incorporated in the recommendation of the guidance.

(ii) The guidance defines an API as "highly soluble" when the highest dose recommended by WHO is soluble in $250 \mathrm{~mL}$ or less of aqueous media over the $\mathrm{pH}$ range of 1.2-6.8. So, with respect to the definition of "highly soluble", the WHO follows the EU approach.

(iii) The guidance defines an API as "highly permeable" when the extent of absorption in humans is $\geq 85 \%$. The guidance FDA, BCS (2000) requires $\geq 90 \%$.

(iv) The guidance allows IR drug products containing BCS class 2 APIs to be considered for a biowaiver, provided that API is a weak acid and meets dose:solubility ratio of $250 \mathrm{~mL}$ or less at $\mathrm{pH}$ 6.8; provided the drug product dissolves rapidly at $\mathrm{pH} 6.8$ ànd provided the risk of reaching an inappropriate biowaiver decision and also the risks to public health and for individual patients is deemed by the national authorities to be acceptable.

(v) The guidance allows IR drug products containing APIs belonging to BCS Class 3 to be considered for a biowaiver, if the drug product and comparator both

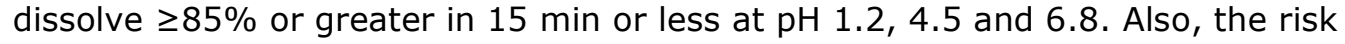
of reaching an inappropriate biowaiver decision and the risks to public health and for individual patients must be deemed acceptable by the national authorities.

(vi) The guidance defines the qualification needed to demonstrate that each excipient does not affect absorption: 
European Journal of Pharmaceutical Sciences Volume 29, Issues 3-4, November 2006, Pages 315-324 Advances in Understanding Oral Absorption and Delivery of Problem Compounds - Selected Papers from the 3rd World Conference on Drug Absorption, Transport and Delivery

a. the excipient is present in the comparator product, or the excipient is present in a number of other products having a MA in ICH or associated countries, and

b. the excipient is present in an amount similar to that in the comparator, or the excipient is present in the multisource drug product in an amount typically used for that type of dosage form.

(vii) The guidance has wider limits for dose proportionally for high potency APIs than the guidance FDA, BA/BE (2003). The FDA accepts qualification for up to $5 \mathrm{mg}$ API per dosage unit, the WHO up to $10 \mathrm{mg}$ per dosage unit.

\section{Biowaivers in practice}

\subsection{Case study 1: formulation development for new drug product + proportionally similar line extension}

\subsubsection{Pharmaceutical situation}

During development of a new oral API for diabetes, qualification was sought for the proposed commercial $5 \mathrm{mg}$ tablet versus the $5 \mathrm{mg}$ prototype tablet. Differences between the two formulations existed only in the amount of lactose and in the presence of colorants. In addition, a biowaiver was pursued for a commercial $2.5 \mathrm{mg}$ tablet, this strength being proportionally similar to the $5 \mathrm{mg}$ commercial tablet, in fact from a common granulation. ${ }^{1}$

\subsubsection{Data}

The API showed pH dependent solubility with very low solubility in acid. The permeability was evaluated in Caco-2 monolayers and the permeability coefficient was higher than the references metoprolol and propranolol, both at $\mathrm{pH} 5.5$ and 7.4. The API was subsequently classified as belonging to BCS Class 2 . Since the solubility was low at $\mathrm{pH} 1.2$ and 4.5 , similarity factors at that $\mathrm{pH}$ values were not considered critical and comparative in vitro dissolution data were generated in $\mathrm{pH}$ 6.8 only. The 2.5 and $5 \mathrm{mg}$ commercial tablets showed dissolution profile similarity with an $f_{2}$ value of 67 at $\mathrm{pH}$ 6.8. The $f_{2}$ value between the prototype and commercial $5 \mathrm{mg}$ tablet was 49 , i.e. below the critical value of 50 .

\subsubsection{Outcome}

The qualification of the $5 \mathrm{mg}$ commercial tablet was accepted by FDA, despite the lack of comparative dissolution testing at $\mathrm{pH} 1.2$ and 4.5, accepting the absence of results that are scientifically irrelevant. As the guidance WHO, Multisource (2005a), accepts biowaiving of APIs belonging to BCS Class 2 under certain conditions, this qualification is concordant with the WHO Guidances also. In the EU, no MA was applied for, but according to the EU regulations, the qualification by biowaiving of the $5 \mathrm{mg}$ commercial tablet would not pass, as the EU does not allow biowaiving for APIs BCS 2 for formulation differences. Also, the therapeutic indication: oral diabetic, will most probably make this biowaiver not acceptable in the EU. Since this API is not highly soluble and changes were made during development, under the Japanese guidelines the biowaiver approach would need to be justified on a scientific basis.

The qualification of the $2.5 \mathrm{mg}$ commercial tablet was accepted by the FDA also, per common granulation. In the EU, no authorization was applied for, but the qualification per common granulation is expected to be acceptable in the EU also. 
European Journal of Pharmaceutical Sciences Volume 29, Issues 3-4, November 2006, Pages 315-324 Advances in Understanding Oral Absorption and Delivery of Problem Compounds - Selected Papers from the 3rd World Conference on Drug Absorption, Transport and Delivery

It is anticipated that a waiver may be possible with appropriate scientific justification and consultation in Japan, since this is a proportional change. The qualification is also concordant with the guidance WHO, Multisource (2005a).

\subsection{Case study 2: new generic}

\subsubsection{Pharmaceutical situation}

Qualification was sought for 80 and $160 \mathrm{mg}$ generic IR tablets $A$ and $B$, containing sotalol $\mathrm{HCl}$, an antiarrhythmic API, versus the same strengths of the innovator Sotalex ${ }^{\circledR}$, tablets C and D (Alt et al., 2004 and Rote Liste, 2004). The qualitative composition of the four tablets is shown in Table 4.

\subsubsection{Data}

The solubility of the API was determined according to the guidance EMEA, BA/BE (2001). The literature indicates this API to be rapidly absorbed with a BA of $90 \%$. The permeability was studied using Caco-2 and the in vitro as well as in vivo data suggested high permeability of this API, and sotalol $\mathrm{HCl}$ was classified as BCS Class 1. Comparative in vitro dissolution profiles of the two generic formulations and appropriate reference products were obtained in $0.1 \mathrm{~N} \mathrm{HCl}$ and in $\mathrm{pH} 4.5$ and 6.8 phosphate buffers in the paddle apparatus at $75 \mathrm{rpm}$. All tablets dissolved $>85 \%$ in $15 \mathrm{~min}$ in all media. It was further argued that the excipients present in the generic tablets are well established and present in amounts in common use in IR tablets. Special attention was paid to mannitol, present in about $50 \%$ of the total tablet weight, as according to a cited reference, mannitol accelerates the small intestine transit time at concentrations of above $0.755 \mathrm{~g}$ per $200 \mathrm{~mL}$, leading to a lower absorption. But the concentration of mannitol in the generic tablets is below one-third of the above-mentioned concentration and it was considered unlikely that mannitol would affect the small intestine transit time in that concentration.

\subsubsection{Outcome}

The German Health authorities accepted this biowaiver, being in compliance with the guidance EMEA, BA/BE (2001). In the USA, no MA was applied for, but this qualification is expected to be acceptable in the USA also. The qualification complies also with the guidance WHO, Multisource (2005a). In Japan, no MA was applied for, but, as biowaiving of new generic drug products is not acceptable in Japan, it is expected that this biowaiver would not be granted in that country.

\section{Experience with biowaivers to date and future trends}

Amidon et al. (1995) introduced the BCS framework 11 years ago, and 5 years later the FDA was the first health authority to adopt this concept. As the EU also adopted this concept a year later, nearly unchanged, it could easily be concluded that BCS was destined to be a great success. Indeed, although solid data are lacking in the open literature, the BCS concept seems to have been used successfully in the field of variations to existing drug products. Benefits of BCSbased biowaivers have, however, not yet been realized to the extent that may have been anticipated for new generic drug products falling outside the 'proportional similarity' concept. The reason for this apparent reluctance to use biowaivers is not entirely clear. It might be that in the USA and the EU few new generic drug products containing an API belonging to BCS Class 1 are developed. But it may also be that pharmaceutical companies do not want to risk rejection of 
European Journal of Pharmaceutical Sciences Volume 29, Issues 3-4, November 2006, Pages 315-324 Advances in Understanding Oral Absorption and Delivery of Problem Compounds - Selected Papers from the 3rd World Conference on Drug Absorption, Transport and Delivery

their request for a biowaiver and thus proceed to carry out an in vivo BE study even when the biowaiver would be a viable option. It may also be that biowaiver is acceptable in one country but not in another, for instance in Japan, forcing the pharmaceutical company to carry out an in vivo BE study anyway. Regardless of the reasons for low usage to this point, the situation may portend two important changes for the future: relaxation of criteria for biowaiver where scientifically possible and global harmonization of the regulations.

Over the years, relaxation of the BCS-biowaiver regulations has been proposed. Some made a plea for setting wider boundaries for BCS Class 1, or revising its wording, allowing biowaivers also for present BCS Class 2/3 APIs or sub-sections of these two classes, called intermediate BCS Classes. For instance, for BCS Class 1 , Yu et al. (2002) proposed to reduce the high permeability requirement for biowaiver from 90 to $85 \%$. An intermediate permeability class with a tentative minimal critical value of $40 \%$ fraction dose absorbed was suggested by Polli et al. (2004) and Blume and Schug (1999) argued that the present BCS Class 3 APIs can be candidates for biowaivers, provided that the drug product is fast dissolving and formulated without excipients which may modify GI transit or membrane permeation, even without setting a minimum value for the permeability.

With respect to relaxing the present boundaries of BCS Class 2 APIs, it was argued that the criterion of solubility of the highest strength in $250 \mathrm{~mL}$ over the range $\mathrm{pH} \mathrm{1-7.5} \mathrm{is} \mathrm{overly} \mathrm{conservative} \mathrm{(Polli} \mathrm{et} \mathrm{al.,} \mathrm{2004);} \mathrm{that} \mathrm{high} \mathrm{solubility}$ should be required only at physiological $\mathrm{pH}$-values; and it was suggested to define an intermediate solubility BCS Class 2 for APIs that are soluble either in the intestine or in the stomach (Yu et al., 2002). Other researchers pointed out that the present BCS system is based on static measurements, whereas the physiological process is dynamic. According to this line of thought, a limited solubility of an API in acid is not physiologically relevant (Rinaki et al., 2004), and the solubility at $\mathrm{pH}>5$ is more appropriate because most compounds are absorbed mainly in the intestinal region (Yazdanian et al., 2004).

Indeed, more relaxed criteria are presented in more recent documents, such as the revised guidance WHO, Multisource (2005a). In view of these developments, the FDA and the EU may also consider revising their guidances, using more relaxed BCS criteria (Polli et al., 2004). Another development in biowaiver regulations that can be expected is the introduction of other in vitro techniques in addition to dissolution testing. There is an increasing awareness that comparative dissolution testing is most relevant only if the solubility of the API or the dissolution of the dosage form in vivo is the rate-limiting step in drug absorption, but is less meaningful if the GI permeability is the rate-limiting step (Blume and Schug, 1999 and Kaus et al., 1999). In this situation, comparative permeability testing may be a valuable adjunct to comparative in vitro dissolution testing as a surrogate methodology for an in vivo BE study. Lastly, it can be hoped and perhaps expected that the criteria and methods for biowaiver will become harmonized on a global level, i.e. ICH-level. This is of utmost importance. Once one country requests an in vivo BE study, this may in effect set the expected standard in other countries; thus, the potential value of biowaiver elsewhere is greatly diminished, even when a biowaiver is fully scientifically valid. This can be a hindrance to timely availability of reliable and affordable medicines, especially in developing countries.

\section{Conclusion}

It is the authors' hope that this brief overview provides a handy quick reference for comparing both the particulars of various guidances on biowaivers for IR oral 
European Journal of Pharmaceutical Sciences Volume 29, Issues 3-4, November 2006, Pages 315-324 Advances in Understanding Oral Absorption and Delivery of Problem Compounds - Selected Papers from the 3rd World Conference on Drug Absorption, Transport and Delivery

solid dosage forms and regional differences in regulatory/scientific philosophy on their applicability. We caution that the regulatory landscape is dynamic, and this review is no substitute for consultation with the full text of currently applicable guidances and regulations, or indeed for direct consultation with the Health Authorities when biowaivers are being considered as part of registrational strategy.

All stakeholders in the development and registration of new drug products must balance the need for scientific rigor in assuring BA/BE (and hence product quality towards consistent therapeutic outcomes) with the time and expense of conducting in vivo BE studies, and the overall impact on product costs and timely availability to patients. Prudent risk analysis based on sound biopharmaceutical science must be considered by regulator and formulator alike. It is further hoped that continued research and scientific/regulatory dialog might lead to optimal and harmonized application of biowaiver principles in the interest of improved worldwide access to needed medicines.

\section{Note added in proof}

The drafts WHO, Multisource, 2005a and WHO, Biowaiver, 2000c have been published as Appendix 7 and Appendix 8 of: WHO, 2006. WHO Technical Report Series 937 of the WHO EXPERT COMMITTEE ON SPECIFICATIONS FOR PHARMACEUTICAL PREPARATIONS. 2004. http://whqlibdoc.who.int/trs/WHO_TRS_937_eng.pdf 
European Journal of Pharmaceutical Sciences Volume 29, Issues 3-4, November 2006, Pages 315-324 Advances in Understanding Oral Absorption and Delivery of Problem Compounds - Selected Papers from the 3rd World Conference on Drug Absorption, Transport and Delivery

\section{References}

Alt et al., 2004 A. Alt, H. Potthast, Moessinger], B. Sickmuller and H. Oeser, Biopharmaceutical characterization of sotalol-containing oral immediate release drug products, Eur. J. Pharm. Biopharm. 58 (2004) (1), pp. 145-150.

Amidon et al., 1995 G.L. Amidon, H. Lennernas, V.P. Shah and J.R. Crison, A theoretical basis for a biopharmaceutic drug classification: the correlation of in vitro drug product dissolution and in vivo bioavailability, Pharm. Res. 12 (1995) (3), pp. 413-420.

Aoyagi, 2000 N. Aoyagi, Japanese guidance on bioavailability and bioequivalence, Eur. J. Drug Metab. Pharmacokinet. 25 (2000) (1), pp. 28-31.

Aoyagi et al., 2004 N. Aoyagi, H. Morikawa, T. Sonobe, K. Yamamoto, S. Kojima, Y. Hiyama, N. Kaniwa, N. Muranishi and Y. Sakai, Guidelines (Draft) for bioequivalence studies for manufacturing changes of oral solid dosage forms: conventional and enteric-coated products, Iyakuhin Kenkyu 35 (2004) (6), pp. 295-317.

Blume and Schug, 1999 H.H. Blume and B.S. Schug, The biopharmaceutics classification system (BCS): class III drugs-better candidates for BA/BE waiver?, Eur. J. Pharm. Sci. 9 (1999) (2), pp. 117-121.

CFR, 2005 CFR, US Code of Federal Regulations 21CFR320.33. Title 21, vol. 5, Revised April 1, 2005, Food and Drug Administration, Department of Health and Human Services

(2005)http://www.accessdata.fda.gov/scripts/cdrh/cfdocs/cfcfr/CFRSearch.cfm?fr=320.33.

EMEA, 2001 EMEA, Note for Guidance on the Investigation of Bioavailability and Bioequivalence, European Agency for Evaluation of Medicinal Products, Committee for Proprietary Medicinal Products (2001)http://www.emea.eu.int/pdfs/human/ewp/140198en.pdf.

EU, 2003 EU, Notice to Applicants. Guideline on Dossier Requirements for Type IA and Type IB Notifications, European Commission (2003)http://dg3.eudra.org/F2/eudralex/vol-

2/C/VariationTypeIAandIBguideline2003_07.doc.

FDA, 1995 FDA, Guidance for Industry. Immediate Release Solid Oral Dosage Forms. Scale-up and Postapproval Changes: Chemistry, Manufacturing, and Controls, In vitro Dissolution Testing and In vivo Bioequivalence Documentation, US Food and Drug Administration, Center for Drug Evaluation and Research, USA (1995)http://www.fda.gov/cder/guidance/cmc5.pdf.

FDA, 1997 FDA, Extended release oral dosage forms: Development, evaluation, and application of in vitro/in vivo correlations, Food and Drug Administration, Center for Drug Evaluation and Research (1997)http://www.fda.gov/cder/guidance/1306fnl.pdf.

FDA, 2000 FDA, Guidance for Industry: Waiver of in vivo Bioavailability and Bioequivalence Studies for Immediate-Release Solid Oral Dosage Forms Based on a Biopharmaceutics Classification System, US Food and Drug Administration, Center for Drug Evaluation and Research, USA (2000)http://www.fda.gov/cder/guidance/3618fnl.pdf.

FDA, 2003 FDA, Guidance for Industry: Bioavailability and Bioequivalence Studies for Orally Administered Drug Products-General Considerations, US Food and Drug Administration Center for Drug Evaluation and Research (2003)http://www.fda.gov/cder/guidance/5356fnl.pdf.

Kaus et al., 1999 L.C. Kaus, W.R. Gillespie, A.S. Hussain and G.L. Amidon, The effect of in vivo dissolution, gastric emptying rate, and intestinal transit time on the peak concentration and areaunder-the-curve of drugs with different gastrointestinal permeabilities, Pharm. Res. 16 (1999) (2), pp. 272-280.

NIHS, 1997 NIHS, Guideline for Bioequivalence Studies of Generic Products, National Institute of Health Sciences, Japan (1997)http://www.nihs.go.jp/drug/be-guide(e)/Generic/be97E.html.

NIHS, 2000a NIHS, Guideline for Bioequivalence Studies for Different Strengths of Oral Solid Dosage Forms, National Institute of Health Sciences, Japan (2000)http://www.nihs.go.jp/drug/beguide(e)/strength/strength.PDF. 
European Journal of Pharmaceutical Sciences Volume 29, Issues 3-4, November 2006, Pages 315-324 Advances in Understanding Oral Absorption and Delivery of Problem Compounds - Selected Papers from the 3rd World Conference on Drug Absorption, Transport and Delivery

NIHS, 2000b NIHS, Guideline for Bioequivalence Studies for Formulation Changes of Oral Solid Dosage Forms, National Institute of Health Sciences, Japan (2000)http://www.nihs.go.jp/drug/beguide(e)/form/form-change.PDF.

Polli et al., 2004 Polli, J.E., Yu, L.X., Cook, J.A., Amidon, G.L., Borchardt, R.T., Burnside, B.A., Burton, P.S., Chen, M.L., Conner, D.P., Faustino, P.J., Hawi, A.A., Hussain, A.S., Joshi, H.N., Kwei, G., Lee, V.H., Lesko, L.J., Lipper, R.A., Loper, A.E., Nerurkar, S.G., Polli, J.W., Sanvordeker, D.R., Taneja, R., Uppoor, R.S., Vattikonda, C.S., Wilding, I., Zhang, G., 2004. Summary Workshop Report:

Biopharmaceutics Classification System-Implementation Challenges and Extension Opportunities. J. Pharm. Sci. 93(6), pp. 1375-1381.

Rinaki et al., 2004 E. Rinaki, A. Dokoumetzidis, G. Valsami and P. Macheras, Identification of biowaivers among Class II drugs: theoretical justification and practical examples, Pharm. Res. 21 (2004) (9), pp. 1567-1572.

Rote Liste, 2004 Rote Liste ${ }^{\circledR}, 2004$. Rote Liste ${ }^{\circledR}$ Service GmbH FMG. Arzneimittelverzeichnis fur Deutschland, in: Verlag EG (Ed.). Aulendorf, Germany. Assessed 2004. http://www.rote-liste.de (after registration).

WHO, 1996 WHO, 1996. Multisource (Generic) Pharmaceutical Products: Guidelines on Registration Requirements to Establish Interchangeability. Technical Report Series No. 863, Annex 9, pp. 115-139. http://whqlibdoc.who.int/trs/WHO_TRS_863_(p99-p194).pdf.

WHO, 2005a WHO 2005a. Multisource (Generic) Pharmaceutical Products: Guidelines on Registration Requirements to Establish Interchangeability. Working document AS/04.093/Rev.4. World Health Organization.

http://www.who.int/medicines/services/expertcommittees/pharmprep/QAS04_093Rev4_final.pdf.

WHO, 2005b WHO, WHO Model List of Essential Medicines (14th ed.), World Health Organization (2005)http://whqlibdoc.who.int/hq/2005/a87017_eng.pdf.

WHO, 2005c WHO, 2005c. Proposal to waive in vivo bioequivalence requirements for the WHO Model List of Essential Medicines immediate release, solid oral dosage forms. Working document QAS/04.109/Rev.1. World Health Organization.

http://www.who.int/medicines/services/expertcommittees/pharmprep/QAS04_109Rev1_Waive_invivo _bioequiv.pdf.

Yazdanian et al., 2004 M. Yazdanian, K. Briggs, C. Jankovsky and A. Hawi, The "high solubility" definition of the current FDA guidance on biopharmaceutical classification system may be too strict for acidic drugs, Pharm. Res. 21 (2004) (2), pp. 293-299.

Yu et al., 2002 L.X. Yu, G.L. Amidon, J.E. Polli, H. Zhao, M.U. Mehta, D.P. Conner, V.P. Shah, L.J. Lesko, M.L. Chen, V.H. Lee and A.S. Hussain, Biopharmaceutics classification system: the scientific basis for biowaiver extensions, Pharm. Res. 19 (2002) (7), pp. 921-925. 